Review

\title{
Long-term clinical and radiographic evaluation of the effectiveness of direct pulp capping materials: A meta-analysis
}

\author{
Takashi MATSUURA¹, S. M. ZIAUDDIN¹, Viviane K. S. KAWATA-MATSUURA², Kouji SUGIMOTO'1, \\ Shizuka YAMADA ${ }^{1}$ and Atsutoshi YOSHIMURA ${ }^{1}$
}
${ }^{1}$ Department of Periodontology and Endodontology, Nagasaki University Graduate School of Biomedical Sciences, 1-7-1, Sakamoto, Nagasaki 852- 8588, Japan
${ }^{2}$ Basic and Translational Research Center for Hard Tissue Disease, Nagasaki University Graduate School of Biomedical Sciences, 1-7-1, Sakamoto, Nagasaki 852-8588, Japan
Corresponding author, Takashi MATSUURA; E-mail: matsuurat@nagasaki-u.ac.jp

\begin{abstract}
The aim of this review is to evaluate the long-term effectiveness of calcium silicate-based cement (CS) and calcium hydroxide (CH) for direct pulp capping (DPC) to human pulp-exposed permanent teeth. An electronic search and manual search were performed on 21 June 2019. Long-term clinical and radiographic evaluations of the effectiveness of CS and CH for DPC to human pulp-exposed teeth were included, and data extraction, risk-of-bias assessment and meta-analyses were performed. From 645 identified articles, 7 articles met the eligibility criteria. The meta-analyses comparing CS with $\mathrm{CH}$ and Biodentine with mineral trioxide aggregates (MTA) on DPC success rate were performed, and significant difference was observed between CS and $\mathrm{CH}$ (risk ratio=1.20; $p=0.005$ ), whereas no significant difference was observed between Biodentine and MTA. CS seems to be a more effective and predictable DPC material than $\mathrm{CH}$; however, these analyses are based on the studies judged at high risk of bias.
\end{abstract}

Keywords: Meta-analysis, Direct pulp capping, Calcium hydroxide, Mineral trioxide aggregate

\section{INTRODUCTION}

Numbers of materials for direct pulp capping (DPC) to human teeth which has exposed dental pulp has been studied; however, lots of studies are basic research such as animal studies and in vitro studies, or clinical studies which do not have control group or evaluate short-term outcome. They are not adequate to evaluate long-term clinical effectiveness of DPC materials; therefore, we performed systematic review to evaluate the long-term effectiveness of DPC materials to pulp-exposed human teeth $^{1}$. Previous study revealed that calcium hydroxide (CH) and calcium silicate-based cement (CS) which includes ProRoot MTA (Dentsply, Tulsa, OK, USA), white ProRoot MTA (WMTA; Dentsply), MTA-Angelus (Angelus Soluções Odontológicas, Londrina, Brazil), Endocem (Maruchi, Wonju, Korea) and Biodentine (Septodont, Saint-Maur-des-Fossés, France) were used on more than 10 randomized clinical trials (RCTs), and the range of the success rate of CS was narrower than that of CH. CS is likely to be more effective for DPC than $\mathrm{CH}$. Therefore, we have following question.

\section{Question 1: "Is CS statistically superior to $\mathrm{CH}$ on long-term clinical and radiographic success rate of DPC to pulp-exposed human permanent teeth?".}

Moreover, previous study revealed that Biodentine was used on $3 \mathrm{RCT}$ s and the success rate was remarkably

Color figures can be viewed in the online issue, which is available at J-STAGE.

Received Feb 10, 2020: Accepted Apr 3, 2020

doi:10.4012/dmj.2020-043 JOI JST.JSTAGE/dmj/2020-043 high (96.4-100\%). Mineral trioxide aggregate (MTA) is a CS based on the Portland cement which contains unpurifiable mixtures of calcium silicates, calcium aluminate, calcium aluminoferrite and calcium sulfate; meanwhile, Biodentine is a CS based on high purity of calcium silicates which was synthesized to get high mechanical strength and short setting times. Therefore, we have following question.

\section{Question 2: "Is Biodentine statistically superior to MTA on long-term clinical and radiographic success rate of DPC to pulp-exposed human permanent teeth?"}

These two questions addressed according to the participants, intervention, control and outcome (PICO) principle were described in Table 1 . The aim of this review is to evaluate the long-term effectiveness of CS and $\mathrm{CH}$ for DPC to human pulp-exposed permanent teeth by meta-analyses comparing $\mathrm{CS}$ with $\mathrm{CH}$ and Biodentine with MTA on long-term DPC success rate.

\section{MATERIALS AND METHODS}

Search strategy, study selection, data extraction and risk of bias assessment

An electronic search was performed on 21 June 2019 in 6 databases (PubMed, Google Scholar, Scopus, The Cochrane Library, ProQuest and EBSCOhost). The search terms used are mentioned in Table 2. Furthermore, the issues of the Journal of Dental Research, Journal of Endodontic, International Endodontic Journal, Journal 
Table 1 PICO questions

\begin{tabular}{lll}
\hline & \multicolumn{1}{c}{ Question 1 } & \multicolumn{1}{c}{ Question 2 } \\
\hline P (Participants) & $\begin{array}{l}\text { pulp-exposed human permanent teeth } \\
\text { with or without caries }\end{array}$ & $\begin{array}{l}\text { pulp-exposed human permanent teeth } \\
\text { with or without caries }\end{array}$ \\
I (Intervention) & DPC with CS & DPC with Biodentine \\
C (Control) & DPC with CH & DPC with MTA \\
O (Outcome) & $\begin{array}{l}\text { clinical and radiographic success rate } \\
\text { with long-term follow-up }\end{array}$ & $\begin{array}{l}\text { clinical and radiographic success rate } \\
\text { with long-term follow-up }\end{array}$ \\
\hline
\end{tabular}

DPC: Direct pulp capping; CS: Calcium silicate-based cement; MTA: Mineral trioxide aggregate; CH: Calcium hydroxide

Table 2 The search strategy used in PubMed (MedLine)

\begin{tabular}{clr}
\hline No. & \multicolumn{1}{c}{ Search terms } & No. of articles \\
\hline$\# 1$ & "dental pulp capping” [MeSH] OR "pulp capping” [TW] & 2,523 \\
$\# 2$ & "randomized" [TW] OR "randomised” [TW] & 825,527 \\
$\# 3$ & $\# 1$ AND \#2 & 192 \\
\hline
\end{tabular}

Table 3 The eligibility criteria

\begin{tabular}{ll}
\multicolumn{1}{c}{ Inclusion criteria } & \multicolumn{1}{c}{ Exclusion criteria } \\
\hline $\begin{array}{l}\text { Language: English } \\
\text { Pulp exposed permanent teeth } \\
\text { with or without caries }\end{array}$ & Not Randomized Clinical Trials \\
Efficacy of pulp-capping materials & Indirect pulp capping, Pulpotomy \\
Clinical and radiographic evaluation & Animal studies, In vitro studies \\
Studies comparing CH with CS & Follow-up period: less than 6 months \\
& $\begin{array}{l}\text { Studies comparing test group } \\
\text { with negative control group }\end{array}$ \\
Studies comparing Biodentine with MTA & $\begin{array}{l}\text { Studies comparing the same material } \\
\text { with different pretreatments }\end{array}$ \\
\hline
\end{tabular}

CH: Calcium hydroxide; CS: Calcium silicate-based cement; MTA: Mineral trioxide aggregate

of Conservative Dentistry and American Journal of Dentistry published until June 2019 (the last issue available) were manually searched.

The eligibility criteria are described in Table 3. Clinical and radiographic studies with long-term follow-up period that assessed the effectiveness of DPC materials in the treatment of human permanent teeth with exposed dental pulp with or without caries were included; moreover only randomized clinical trials (RCTs) with follow-up periods of more than 6 months were included, because RCT is a most convincing study design and a less than 3-months follow-up period would not be sufficient to guarantee the long-term prognosis of a DPC material.

The titles and abstracts of the articles were screened for a full-text evaluation, and duplicates, reviews and non-English articles were removed. The full texts were screened for eligibility, and only articles met all of the eligibility criteria were included in this study and processed for data extraction.
Data extraction and risk of bias assessment were performed as described before ${ }^{1)}$. Literature search, study selection, data extraction and risk of bias assessment were independently performed by two reviewers (S.M.Z. and V.K.S.K.).

\section{Statistical analysis}

All statistical analyses were performed using Review Manager (RevMan) version 5.3.5. (Cochrane Collaboration software). Risk ratio (RR) and 95\% confidence interval (CI) were used to compare the success rate of $\mathrm{CS}$ with that of $\mathrm{CH}$ and the success rate of Biodentine with that of MTA. Clinical and methodological heterogeneities among included studies were observed; therefore, combined RRs were calculated using the random effect model for meta-analysis. The Cochrane $\mathrm{Q}$ test and the $\mathrm{I}^{2}$ statistic were used to test heterogeneity. Publication bias assessment were evaluated with funnel plots in RevMan 5. 
Table 4 The characteristics of the included studies (part 1)

\begin{tabular}{|c|c|c|c|c|c|c|c|c|}
\hline \multirow[b]{2}{*}{$\begin{array}{l}\text { Author } \\
\text { (year) } \\
\text { Ref.) }\end{array}$} & \multicolumn{2}{|c|}{ Participants } & \multirow[b]{2}{*}{$\begin{array}{l}\text { Types of } \\
\text { teeth }\end{array}$} & \multirow[b]{2}{*}{$\begin{array}{l}\text { Deep } \\
\text { caries }\end{array}$} & \multirow[b]{2}{*}{$\begin{array}{l}\text { Pulp exposure } \\
\text { size in diameter }\end{array}$} & \multirow[b]{2}{*}{$\begin{array}{l}\text { Bleeding } \\
\text { controlled within }\end{array}$} & \multirow[b]{2}{*}{$\begin{array}{c}\text { Group (n) } \\
\text { [test (T) and control }(\mathrm{C})]\end{array}$} & \multirow[b]{2}{*}{$\begin{array}{l}\text { No. of } \\
\text { ope- } \\
\text { rators }\end{array}$} \\
\hline & No. & $\begin{array}{l}\text { Age mean } \\
\text { (range) } \\
\text { in years } \\
\end{array}$ & & & & & & \\
\hline $\begin{array}{l}\text { Awawdeh } \\
\text { et al. (2018) } \\
\text { 2) }\end{array}$ & 58 & $32.5(16-51)$ & $\begin{array}{l}\text { permanent } \\
\text { teeth }\end{array}$ & + & $\begin{array}{l}\text { NA } \\
\text { (created with a } \\
\text { new round } \\
\text { bur ISO no.23) }\end{array}$ & $3 \min$ & $\begin{array}{l}\text { T: Biodentine (11) } \\
\text { C: MTA-Angelus (6) }\end{array}$ & NA \\
\hline $\begin{array}{l}\text { Kundzina } \\
\text { et al. }(2017)\end{array}$ & 70 & $30.6(18-55)$ & $\begin{array}{l}\text { 1st or 2nd } \\
\text { permanent } \\
\text { molar }\end{array}$ & + & NA & $10 \mathrm{~min}$ & $\begin{array}{l}\text { T: WMTA (33) } \\
\text { C: CH (Dycal) (37) }\end{array}$ & 6 \\
\hline $\begin{array}{l}\text { Hilton } \\
\text { et al. }(2013) \\
\text { 6) }\end{array}$ & 376 & $37.9(8-90)$ & $\begin{array}{l}\text { permanent } \\
\text { teeth }\end{array}$ & $\begin{array}{l}\text { Trauma } \\
\text { or } \\
\text { caries }\end{array}$ & $\begin{array}{l}\text { Less than } 1 \mathrm{~mm} \\
{[87.5 \%(\mathrm{MTA}), 81.8 \%} \\
(\mathrm{CH})] \\
\text { More than } 1.5 \mathrm{~mm} \\
{[12.6 \% \text { (MTA), } 18.3 \%} \\
(\mathrm{CH})]\end{array}$ & $\begin{array}{l}\text { until bleeding } \\
\text { controlled or } \\
\text { operator decided } \\
\text { DPC not } \\
\text { appropriate }\end{array}$ & $\begin{array}{l}\text { T: ProRoot MTA (195) } \\
\text { CH (Life) (181) }\end{array}$ & 35 \\
\hline $\begin{array}{l}\text { Parinyaprom } \\
\text { et al. (2017) } \\
\text { 5) }\end{array}$ & $\mathrm{NA}$ & NA $(6-18)$ & $\begin{array}{l}\text { permanent } \\
\text { teeth }\end{array}$ & + & Less than $2.5 \mathrm{~mm}$ & $10 \mathrm{~min}$ & $\begin{array}{l}\text { T: Biodentine (29) } \\
\text { C: ProRoot MTA (30) }\end{array}$ & 8 \\
\hline $\begin{array}{l}\text { Brizuela } \\
\text { et al. (2017) } \\
\text { 3) }\end{array}$ & 169 & $11.3(7-16)$ & $\begin{array}{l}\text { permanent } \\
\text { teeth }\end{array}$ & + & Less than $2 \mathrm{~mm}$ & $10 \mathrm{~min}$ & $\begin{array}{l}\text { T1: Biodentine (60) } \\
\text { T2: WMTA (56) } \\
\text { C: CH capsule (53) }\end{array}$ & 5 \\
\hline $\begin{array}{l}\text { Katge } \\
\text { et al. }(2017) \\
\text { 7) }\end{array}$ & 50 & NA (7-9) & $\begin{array}{l}1 \text { st } \\
\text { permanent } \\
\text { molar }\end{array}$ & + & Less than $1 \mathrm{~mm}$ & $\mathrm{NA}$ & $\begin{array}{l}\text { T: Biodentine (29) } \\
\text { C: MTA-Angelus (29) }\end{array}$ & 1 \\
\hline $\begin{array}{l}\text { Suhag } \\
\text { et al. }(2019) \\
\text { 4) }\end{array}$ & 64 & $21.8(15-40)$ & $\begin{array}{l}\text { 1st or 2nd } \\
\text { permanent } \\
\text { molar }\end{array}$ & + & NA & $10 \mathrm{~min}$ & $\begin{array}{l}\text { T: WMTA (32) } \\
\text { C: CH (powder) (32) }\end{array}$ & 1 \\
\hline
\end{tabular}

CH: Calcium hydroxide; DPC: Direct pulp capping; MTA: Mineral Trioxide Aggregate; NA: not available; WMTA: white ProRoot MTA

The characteristics of the included studies (part 2)

\begin{tabular}{|c|c|c|c|c|c|c|c|}
\hline \multirow[b]{2}{*}{$\begin{array}{c}\text { Author (year) } \\
\text { Ref.) }\end{array}$} & \multirow[b]{2}{*}{$\begin{array}{l}\text { Rubber } \\
\text { dam } \\
\text { isolation }\end{array}$} & \multirow[b]{2}{*}{$\begin{array}{l}\text { Disinfection } \\
\text { or hemostasis } \\
\text { with } \mathrm{NaOCl}\end{array}$} & \multirow[b]{2}{*}{$\begin{array}{l}\text { Final } \\
\text { restoration }\end{array}$} & \multirow[b]{2}{*}{$\begin{array}{l}\text { Final } \\
\text { follow-up } \\
\text { (months) }\end{array}$} & \multicolumn{2}{|c|}{ At final follow-up } & \multirow[b]{2}{*}{$\begin{array}{l}\text { Statistical } \\
\text { analysis }\end{array}$} \\
\hline & & & & & $\begin{array}{l}\text { Recall rate } \\
\quad(\mathrm{n} / \mathrm{N})\end{array}$ & $\begin{array}{c}\text { Clinical and } \\
\text { radiographic success rate } \\
\text { (success/total) [test (T) } \\
\text { and control (C)] }\end{array}$ & \\
\hline $\begin{array}{l}\text { Awawdeh } \\
\text { et al. }(2018) \\
\text { 2) }\end{array}$ & + & + & $\begin{array}{l}\mathrm{AM} \\
\mathrm{CR}\end{array}$ & 36 & $\begin{array}{l}\text { T: } 90.9 \%(10 / 11) \\
\text { C: } 83.3 \%(5 / 6)\end{array}$ & $\begin{array}{l}\mathrm{T}: 90 \%(9 / 10) \\
\mathrm{C}: 100 \%(5 / 5)\end{array}$ & $\begin{array}{l}\text { No } \\
\text { significant } \\
\text { difference }\end{array}$ \\
\hline $\begin{array}{l}\text { Kundzina } \\
\text { et al. }(2017) \\
\text { 8) }\end{array}$ & \pm & + & $\mathrm{CR}$ & 36 & $\begin{array}{l}\text { T: } 93.9 \%(31 / 33) \\
\text { C: } 91.9 \%(34 / 37)\end{array}$ & $\begin{array}{l}\text { T: } 85 \% \text { (NA) } \\
\text { C: } 52 \% \text { (NA) }\end{array}$ & $\begin{array}{l}\text { Significant } \\
\text { difference }\end{array}$ \\
\hline $\begin{array}{l}\text { Hilton } \\
\text { et al. (2013) } \\
\text { 6) }\end{array}$ & \pm & $\begin{array}{l}92.3 \% \text { (MTA group) } \\
82.9 \% \text { (CH group) }\end{array}$ & $\begin{array}{l}\text { None }(36.9 \%) \\
\text { AM }(35.2 \%) \\
\text { CR }(21.5 \%) \\
\text { Other }(6.4 \%)\end{array}$ & 24 & $\begin{array}{l}\text { T: } 93.8 \%(183 / 195) \\
\text { C: } 96.7 \%(175 / 181)\end{array}$ & $\begin{array}{l}\text { Kaplan-Meier estimate } \\
\text { T: } 77.6 \%, \text { C: } 68.5 \% \\
\text { Crude success rate } \\
\text { T: } 86.3 \%(158 / 183) \text {, } \\
\text { C: } 75.4 \%(132 / 175)\end{array}$ & $\begin{array}{l}\text { Significant } \\
\text { difference }\end{array}$ \\
\hline $\begin{array}{l}\text { Parinyaprom } \\
\text { et al. }(2017) \\
\text { 5) }\end{array}$ & + & + & $\begin{array}{l}\text { CR }(70.9 \%) \\
\text { AM }(1.8 \%) \\
\operatorname{SSC}(27.3 \%)\end{array}$ & $\begin{array}{r}18.9 \\
\pm 12.9\end{array}$ & $\begin{array}{l}\text { T: } 96.6 \%(28 / 29) \\
\text { C: } 90 \%(27 / 30)\end{array}$ & $\begin{array}{l}\text { T: } 96.4 \%(27 / 28) \\
\text { C: } 92.6 \%(25 / 27)\end{array}$ & $\begin{array}{l}\text { No } \\
\text { significant } \\
\text { difference }\end{array}$ \\
\hline $\begin{array}{l}\text { Brizuela } \\
\text { et al. }(2017) \\
\text { 3) }\end{array}$ & + & - & $\mathrm{CR}$ & 12 & $\begin{array}{l}\mathrm{T} 1: 41.7 \%(25 / 60) \\
\mathrm{T} 2: 44.6 \%(25 / 56) \\
\text { C: } 47.2 \%(25 / 53)\end{array}$ & $\begin{array}{l}\text { T1: } 100 \%(25 / 25) \\
\text { T2: } 86.36 \%(19 / 22) \\
\text { C: } 86.36 \%(19 / 22)\end{array}$ & $\begin{array}{l}\text { No } \\
\text { significant } \\
\text { difference }\end{array}$ \\
\hline $\begin{array}{l}\text { Katge } \\
\text { et al. }(2017) \\
\text { 7) }\end{array}$ & + & $\begin{array}{l}+ \text { (When bleeding } \\
\text { persisted after } \\
\text { pressure application) }\end{array}$ & $\mathrm{CR}$ & 12 & $\begin{array}{l}\text { T: } 72.4 \%(21 / 29) \\
\text { C: } 72.4 \%(21 / 29)\end{array}$ & $\begin{array}{l}\text { T: } 100 \%(21 / 21) \\
\text { C: } 100 \%(21 / 21)\end{array}$ & $\begin{array}{l}\text { No } \\
\text { significant } \\
\text { difference }\end{array}$ \\
\hline $\begin{array}{l}\text { Suhag } \\
\text { et al. (2019) } \\
\text { 4) }\end{array}$ & + & + & $\mathrm{CR}$ & 12 & $\begin{array}{l}\text { T: } 84.4 \%(27 / 32) \\
\text { C: } 90.6 \%(29 / 32)\end{array}$ & $\begin{array}{l}\text { T: } 93 \%(25 / 27) \\
\text { C: } 69 \%(20 / 29)\end{array}$ & $\begin{array}{l}\text { Significant } \\
\text { difference }\end{array}$ \\
\hline
\end{tabular}

CH: Calcium hydroxide; MTA: Mineral Trioxide Aggregate; AM: Amalgam; CR: Composite resin; SSC: Stainless steel crown 


\section{RESULTS}

\section{Literature identification}

A flowchart summarizing the screening process is presented in Fig. 1. The electronic search retrieved 630 articles; 15 additional articles were found by the hand search. After the first screening, 271 articles were excluded because they were duplicates, reviews or published in a language other than English; 374 articles were assessed for eligibility. After the fulltext evaluation, 367 articles which did not match the eligibility criteria were excluded, and 7 articles were included in this study ${ }^{2-8)}$.

\section{Characteristics of the included studies}

The characteristics of the included studies are presented in Table 4. Four RCTs compared CS with $\mathrm{CH}$ and four RCTs compared Biodentine with MTA. In these included studies, clinical success of DPC was defined as a tooth with normal response to pulp sensitivity tests, no pain, no other clinical symptoms, and no radiographic signs of root resorption and apical periodontitis.

The risk of bias in the included studies is summarized in Table 5. All included studies were judged at high risk of bias.

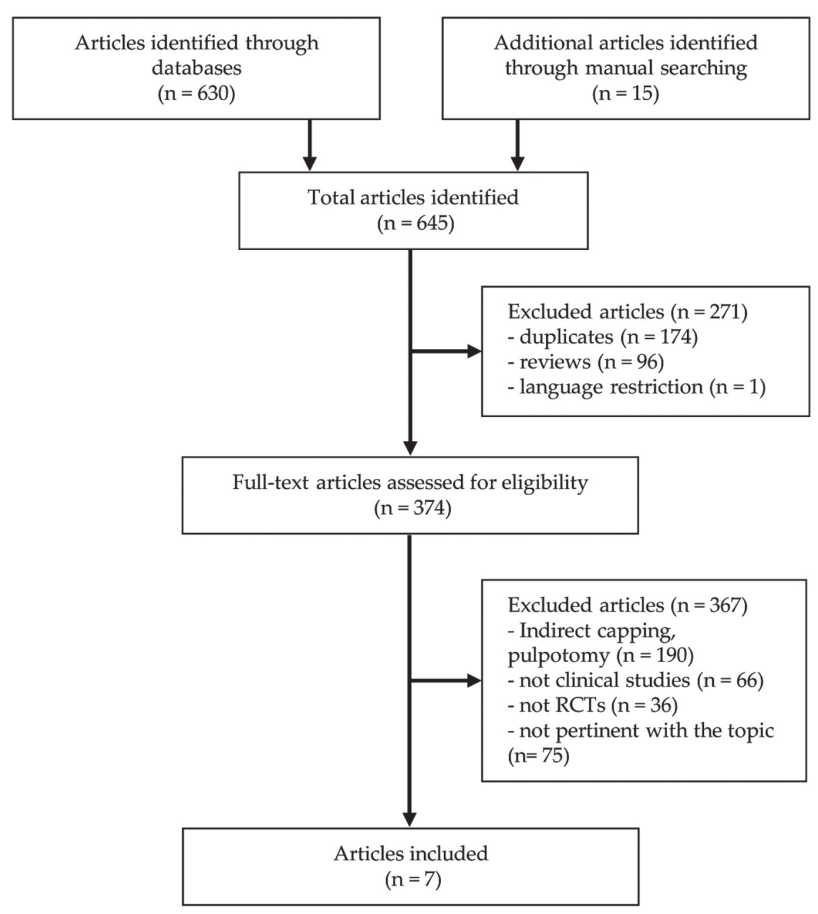

Fig. 1 Flowchart for the meta-analyses.

Table 5 Risk of bias of the included studies

\begin{tabular}{|c|c|c|c|c|c|c|c|c|c|}
\hline \multirow[b]{2}{*}{$\begin{array}{c}\text { Author (year) } \\
\text { Ref.) }\end{array}$} & \multirow{2}{*}{$\begin{array}{l}\text { Random } \\
\text { sequence } \\
\text { generation }\end{array}$} & \multirow{2}{*}{$\begin{array}{l}\text { Allocation } \\
\text { concealment }\end{array}$} & \multicolumn{3}{|c|}{ Blinding of } & \multirow{2}{*}{$\begin{array}{l}\text { Selective } \\
\text { reporting }\end{array}$} & \multirow{2}{*}{$\begin{array}{l}\text { Incomplete } \\
\text { outcome } \\
\text { data }\end{array}$} & \multirow[b]{2}{*}{$\begin{array}{c}\text { Other } \\
\text { bias }\end{array}$} & \multirow[b]{2}{*}{ Overall } \\
\hline & & & patients & operators & $\begin{array}{c}\text { outcome } \\
\text { assessment }\end{array}$ & & & & \\
\hline $\begin{array}{l}\text { Awawdeh } \\
\text { et al. (2018) } \\
\text { 2) }\end{array}$ & $\begin{array}{l}\text { Low } \\
\text { (coin toss) }\end{array}$ & Unclear & Low & Unclear & Low & Low & Low & Low & High \\
\hline $\begin{array}{l}\text { Kundzina } \\
\text { et al. (2017) } \\
\text { 8) }\end{array}$ & $\begin{array}{l}\text { Low } \\
\text { (Envelop } \\
\text { method) }\end{array}$ & $\begin{array}{l}\text { Low } \\
\text { (Central } \\
\text { randomization) }\end{array}$ & Low & High & Low & Low & Low & Low & High \\
\hline $\begin{array}{l}\text { Hilton } \\
\text { et al. (2013) } \\
\text { 6) }\end{array}$ & $\begin{array}{l}\text { Low } \\
\text { (R 2.15) } \\
\text { (Randomization } \\
\text { was done by } \\
\text { practice) }\end{array}$ & $\begin{array}{l}\text { Low } \\
\text { (Central } \\
\text { randomization) }\end{array}$ & Unclear & High & $\begin{array}{l}\text { High } \\
\text { (Operator } \\
\text { assessed } \\
\text { radiograph) }\end{array}$ & Low & Low & Low & High \\
\hline $\begin{array}{l}\text { Parinyaprom } \\
\text { et al. }(2017) \\
\text { 5) }\end{array}$ & $\begin{array}{l}\text { Low } \\
\text { (Randomization } \\
\text { number table) }\end{array}$ & Unclear & Low & High & Low & Low & Low & Low & High \\
\hline $\begin{array}{l}\text { Brizuela } \\
\text { et al. (2017) } \\
\text { 3) }\end{array}$ & $\begin{array}{l}\text { Low } \\
\text { (Excel table) }\end{array}$ & Unclear & Unclear & High & Unclear & Low & $\begin{array}{l}\text { High } \\
\text { (Missing data } \\
\text { can influence } \\
\text { the result) }\end{array}$ & Low & High \\
\hline $\begin{array}{l}\text { Katge } \\
\text { et al. }(2017) \\
\text { 7) }\end{array}$ & Unclear & Unclear & Low & High & Unclear & Low & Low & Low & High \\
\hline $\begin{array}{l}\text { Suhag } \\
\text { et al. (2019) } \\
\text { 4) }\end{array}$ & $\begin{array}{l}\text { Low } \\
\text { (Envelop } \\
\text { method) }\end{array}$ & $\begin{array}{l}\text { Low } \\
\text { (Independent } \\
\text { research } \\
\text { associate) }\end{array}$ & Low & High & Low & Low & Low & Low & High \\
\hline
\end{tabular}

Low: Low risk of bias; Unclear: Unclear risk of bias; High: High risk of bias. 


\section{CS versus $\mathrm{CH}$}

Four RCTs comparing CS with $\mathrm{CH}$ presented in Table 6 were scrutinized in the meta-analysis ${ }^{3,4,6,8}$. White ProRoot MTA was used in 3 RCTs; meanwhile, ProRoot MTA and Biodentine were used in 1 RCT. CH powder (Hertz Pharmaceutical, Santiago, Chile; Prevest DenPro, Jammu, India) and Dycal (Dentsply, Milford, DE, USA) was used in 2 RCTs; meanwhile, Life (Kerr, Orange, CA, USA) was used in $1 \mathrm{RCT}$. The result is presented in Fig. 2. According to the Cochrane Q test and the $\mathrm{I}^{2}$ statistic, the results showed moderate heterogeneity $\left(\mathrm{df}=3 ; p=0.17 ; \mathrm{I}^{2}=40 \%\right)$. The total number of positive events for CS was 253 of 288 DPC treatments, whereas 189 of 260 were recorded for $\mathrm{CH}$. The RR was 1.20 (95\% CI, 1.06 to $1.36 ; p=0.005)$.

\section{Biodentine versus MTA}

Four RCTs comparing Biodentine with MTA presented in Table 7 were scrutinized in the meta-analysis ${ }^{2,3,5,7)}$. MTA-Angelus was used in 2 RCTs; meanwhile, WMTA and ProRoot MTA were used in $1 \mathrm{RCT}$. The result is presented in Fig. 3. According to the Cochrane Q test

Table 6 Summary of the included studies comparing CS with $\mathrm{CH}$

\begin{tabular}{|c|c|c|}
\hline Author (Year) ${ }^{\text {Ref. }}$ & $\mathrm{CH}(\mathrm{n} / \mathrm{N})$ & $\mathrm{CS}(\mathrm{n} / \mathrm{N})$ \\
\hline Brizuela et al. $(2017)^{3)}$ & Powder $(19 / 22)$ & Biodentine (25/25), WMTA (19/22) \\
\hline Hilton et al. $(2013)^{6)}$ & Life $(132 / 175)$ & ProRoot MTA (158/183) \\
\hline Kundzina et al. $(2017)^{8)}$ & Dycal (18/34) & WMTA (26/31) \\
\hline Suhag et al. $(2019)^{4)}$ & Powder (20/29) & WMTA $(25 / 27)$ \\
\hline
\end{tabular}

CH: Calcium hydroxide; CS: Calcium silicate-based cement; WMTA: white ProRoot MTA

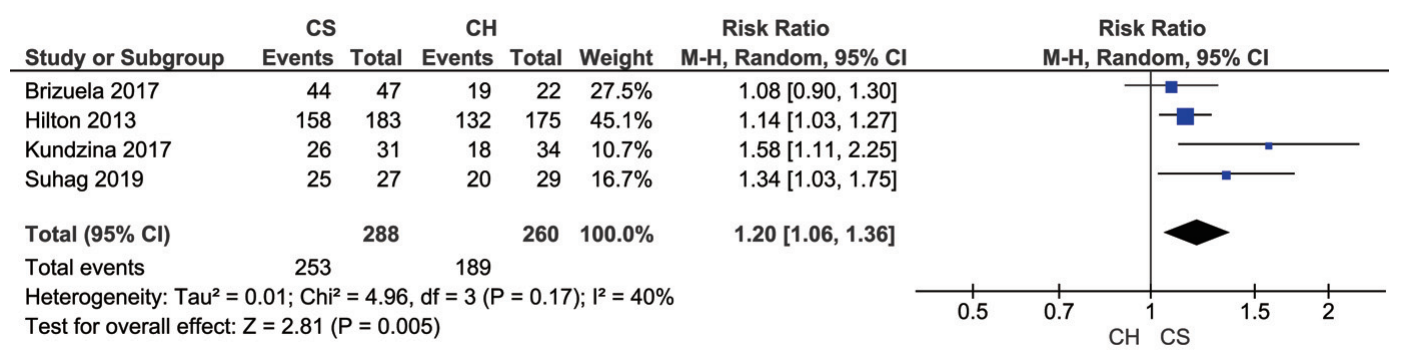

Fig. 2 Forest plot comparing CS with $\mathrm{CH}$ in terms of effectiveness of a DPC material.

Table 7 Summary of the included studies comparing Biodentine with MTA.

\begin{tabular}{lll}
\hline \multicolumn{1}{c}{ Author $(\text { Year })^{\text {Ref. }}$} & \multicolumn{1}{c}{ MTA (n/N) } & Biodentine (n/N) \\
\hline Brizuela et al. $(2017)^{3)}$ & WMTA (19/22) & Biodentine $(25 / 25)$ \\
Katge et al. $(2017)^{7)}$ & MTA angelus (21/21) & Biodentine (21/21) \\
Parinyaprom et al. $(2017)^{5)}$ & ProRoot MTA (25/27) & Biodentine (27/28) \\
Awawdeh et al. $(2018)^{2)}$ & MTA angelus (5/5) & Biodentine (9/10) \\
\hline
\end{tabular}

MTA: Mineral Trioxide Aggregate; WMTA: white ProRoot MTA

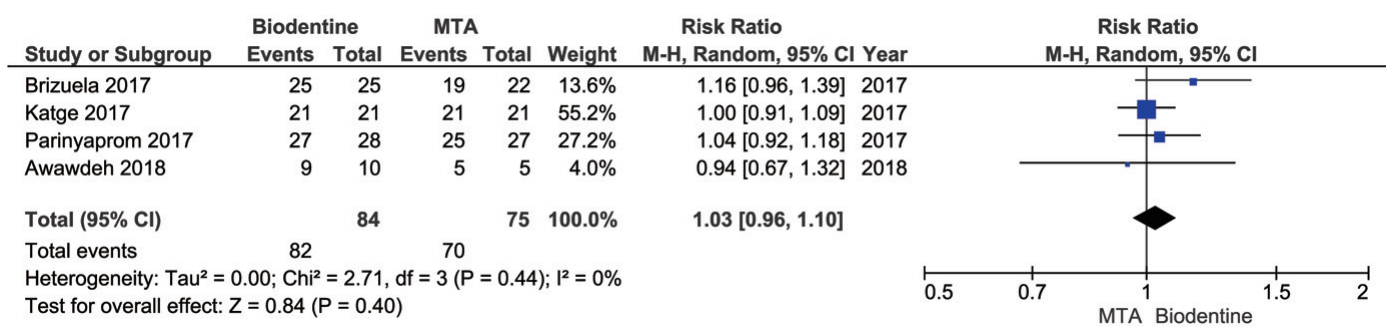

Fig. 3 Forest plot comparing Biodentine with MTA in terms of effectiveness of a DPC material. 


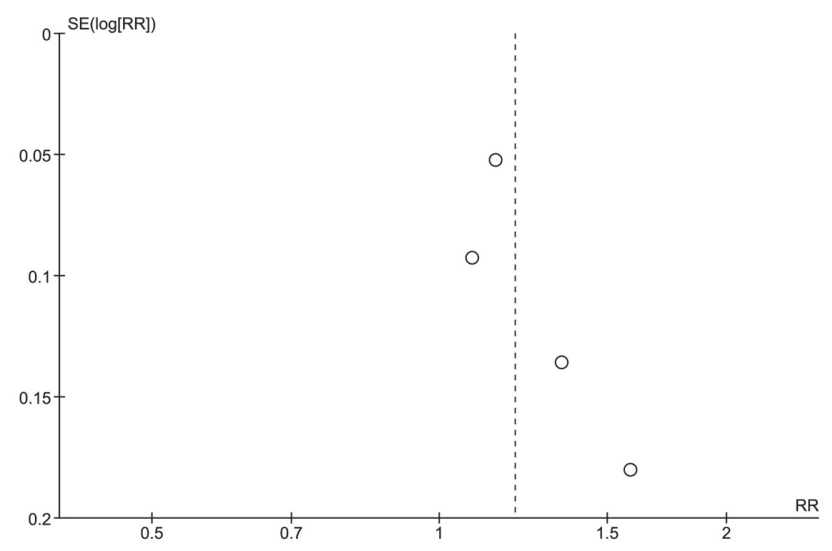

Fig. 4 Funnel plot of meta-analysis comparing CS with $\mathrm{CH}$ (a), and Biodentine with MTA (b).

and the $\mathrm{I}^{2}$ statistic, the results showed no heterogeneity $\left(\mathrm{df}=2.71 ; p=0.44 ; \mathrm{I}^{2}=0 \%\right)$. The total number of positive events for Biodentine was 82 of 84 DPC treatments; whereas, 70 of 75 were recorded for MTA. The RR was 1.03 (95\% CI, 0.96 to $1.10 ; p=0.40)$.

\section{Publication bias assessment}

Publication bias in meta-analyses were assessed by visual inspection of funnel plots shown in Fig. 4. Statistical analyses were not performed because the number of RCTs was small. Asymmetry was not clearly observed in both funnel plots.

\section{DISCUSSION}

This study has some limitations. First, there is a risk of selection bias because only studies in English were included. Second, blinding of operators was impossible because of different clinical handling; therefore, the risk of bias in this domain is unavoidable. Third, only histological analysis can evaluate the true condition of the dental pulp tissue after $\mathrm{DPC}^{\text {9) }}$; however, the followup period of studies in which histological evaluations were performed were short-term, and it is impossible to guarantee the long-term prognosis of a DPC material.

Clinical and methodological heterogeneities of both analyses were not low because of various characteristics and designs of included studies; therefore, statistical heterogeneity was analyzed to know whether it is possible to integrate studies through meta-analyses. The heterogeneity analyses using RevMan 5 indicated that the statistical heterogeneities of both analyses were not serious; therefore, meta-analyses were performed with random effect model, and subgroup analysis was not performed.

Recently, some meta-analyses of studies comparing MTA with CH on DPC success rate to permanent teeth were reported $^{10-12)}$. In 2015 , Zhu et al. compared success rate of MTA with that of $\mathrm{CH}$, and indicated that the success rate of MTA is significantly higher than that of $\mathrm{CH}($ risk difference $=0.1$ [95\% CI, 0.04 to $0.16 ; p=0.002])^{10)}$.
However, this review is unsuitable for the evaluation of MTA and $\mathrm{CH}$ as DPC materials because it included a RCT which evaluated the effectiveness of materials for indirect pulp capping ${ }^{13)}$. Li et al. compared $\mathrm{CH}$ with MTA on success rate, hard-tissue barrier formation and inflammatory response; and their study indicated that the success rate of MTA was significantly higher than that of $\mathrm{CH}$ (odds ratio=2.26 [95\% CI, 1.33 to 3.85 ; $p=0.003])^{11)}$. However, only 2 RCTs were included in this review and one of them was 3-months follow-up study. Lots of studies are performed with less than 6 months follow-up period; however, most clinicians want to know long-term effectiveness. Therefore, studies with less than 6 months follow-up period were excluded in our study. In 2018, Paula et al. compared MTA with $\mathrm{CH}$ on success rate, hard-tissue barrier formation and inflammatory response; and their study indicated that the success rate of MTA was significantly higher than that of $\mathrm{CH}$ (odds ratio $=2.64[95 \% \mathrm{CI}, 1.60$ to $4.35 ; p=0.000])^{12)}$. However, only 2 cohort studies were included and no RCT was included in this meta-analysis. As Cohort study is less convincing than RCT, only RCTs were included in our study.

This study indicates that the success rate of CS is statistically superior to that of $\mathrm{CH}(p=0.005)$; therefore, CS seems to be a more effective and predictable DPC material than $\mathrm{CH}$. Furthermore, it is reported that the long-term cost-effectiveness of MTA for DPC was better than that of $\mathrm{CH}^{14)}$. However, the initial treatment cost of $\mathrm{CS}$ is much higher than that of $\mathrm{CH}$; therefore, clinicians should discuss with patients about choice of a DPC material. Meanwhile, this study also indicates that there was no significant difference between Biodentine and MTA on DPC success rate. And Biodentine is reported to promote high amount of dentine bridge formation in human teeth without any pulpal inflammation similar to white ProRoot MTA ${ }^{15,16)}$. However, other properties of Biodentine such as handling, setting time and tooth discoloration are superior to white ProRoot MTA ${ }^{17)}$. Thus, Biodentine is likely to be more suitable for DPC than white ProRoot MTA.

\section{CONCLUSION}

CS is likely to be a more effective and predictable DPC material than $\mathrm{CH}$, and Biodentine is likely to be more suitable for DPC than white ProRoot MTA. However, data used in this study are based on the included studies judged at high risk of bias, and the statistical analysis was not performed for publication bias assessment because of small number of included RCTs. Therefore, further clinical and radiographic RCTs with long-term follow-up periods are required to confirm which material is a most suitable for DPC.

\section{ACKNOWLEDGMENTS}

Author Contributions: Conceptualization, T.M.; Methodology, T.M.; Formal Analysis, T.M.; Investigation, T.M., Z.S.M. and V.K.S.; Writing-Original Draft 
Preparation, T.M.; Writing-Review \& Editing, K.S. and S.Y.; Visualization, T.M.; Supervision, A.Y.; Project Administration, T.M.

\section{CONFLICTS OF INTEREST}

The authors declare no conflict of interest associated with this research.

\section{REFERENCES}

1) Matsuura T, Kawata-Matsuura VKS, Yamada S. Long-term clinical and radiographic evaluation of the effectiveness of direct pulp-capping materials. J Oral Sci 2019; 61: 1-12.

2) Awawdeh L, Al-Qudah A, Hamouri H, Chakra RJ. Outcomes of vital pulp therapy using mineral trioxide aggregate or Biodentine: a prospective randomized clinical trial. J Endod 2018; 44: 1603-1609.

3) Brizuela C, Ormeno A, Cabrera C, Cabezas R, Silva CI, Ramirez V, et al. Direct pulp capping with calcium hydroxide, mineral trioxide aggregate, and Biodentine in permanent young teeth with caries: a randomized clinical trial. J Endod 2017; 43: 1776-1780.

4) Suhag K, Duhan J, Tewari S, Sangwan P. Success of direct pulp capping using mineral trioxide aggregate and calcium hydroxide in mature permanent molars with pulps exposed during carious tissue removal: 1-year follow-up. J Endod 2019; 45: 840-847.

5) Parinyaprom N, Nirunsittirat A, Chuveera $P$, Na Lampang $\mathrm{S}$, Srisuwan T, Sastraruji T, et al. Outcomes of direct pulp capping by using either ProRoot mineral trioxide aggregate or Biodentine in permanent teeth with carious pulp exposure in 6- to 18-year-old patients: a randomized controlled trial. J Endod 2018; 44: 341-348

6) Hilton TJ, Ferracane JL, Mancl L. Comparison of $\mathrm{CaOH}$ with MTA for direct pulp capping: a PBRN randomized clinical trial. J Dent Res 2013; 92: 16s-22s.

7) Katge FA, Patil DP. Comparative analysis of 2 calcium silicate-based cements (Biodentine and mineral trioxide aggregate) as direct pulp-capping agent in young permanent molars: a split mouth study. J Endod 2017; 43: 507-513.

8) Kundzina R, Stangvaltaite L, Eriksen HM, Kerosuo E. Capping carious exposures in adults: a randomized controlled trial investigating mineral trioxide aggregate versus calcium hydroxide. Int Endod J 2017; 50: 924-932.

9) Hilton TJ. Keys to clinical success with pulp capping: a review of the literature. Oper Dent 2009; 34: 615-625.

10) Zhu C, Ju B, Ni R. Clinical outcome of direct pulp capping with MTA or calcium hydroxide: a systematic review and meta-analysis. Int J Clin Exp Med 2015; 8: 17055-17060.

11) Li Z, Cao L, Fan M, Xu Q. Direct pulp capping with calcium hydroxide or mineral trioxide aggregate: a meta-analysis. J Endod 2015; 41: 1412-1417.

12) Paula AB, Laranjo M, Marto CM, Paulo S, Abrantes AM, Casalta-Lopes J, et al. Direct pulp capping: what is the most effective therapy?-systematic review and meta-analysis. J Evid Based Dent Pract 2018; 18: 298-314.

13) Leye Benoist F, Gaye Ndiaye F, Kane AW, Benoist HM, Farge P. Evaluation of mineral trioxide aggregate (MTA) versus calcium hydroxide cement $(\operatorname{Dycal}((\mathrm{R})))$ in the formation of a dentine bridge: a randomised controlled trial. Int Dent $\mathrm{J}$ 2012; 62: 33-39.

14) Schwendicke F, Brouwer F, Stolpe M. Calcium hydroxide versus mineral trioxide aggregate for direct pulp capping: a cost-effectiveness analysis. J Endod 2015; 41: 1969-1974.

15) Nowicka A, Lipski M, Parafiniuk M, Sporniak-Tutak K, Lichota D, Kosierkiewicz A, et al. Response of human dental pulp capped with biodentine and mineral trioxide aggregate. J Endod 2013; 39: 743-747.

16) Nowicka A, Wilk G, Lipski M, Kolecki J, BuczkowskaRadlinska J. Tomographic evaluation of reparative dentin formation after direct pulp capping with $\mathrm{Ca}(\mathrm{OH}) 2$, MTA, Biodentine, and dentin bonding system in human teeth. J Endod 2015; 41: 1234-1240.

17) Valles M, Roig M, Duran-Sindreu F, Martinez S, Mercade M. Color stability of teeth restored with Biodentine: a 6-month in vitro study. J Endod 2015; 41: 1157-1160. 